\title{
Hitchhiking Through the Cytoplasm
}

\author{
Igor M. Kuliđ and Philip C. Nelson \\ Department of Physics and Astronomy, University of Pennsylvania Philadelphia, PA 19104, USA
}

(Dated: August 20, 2018)

\begin{abstract}
We propose an alternative mechanism for intracellular cargo transport which results from motor induced longitudinal fluctuations of cytoskeletal microtubules (MT). The longitudinal fluctuations combined with transient cargo binding to the MTs lead to long range transport even for cargos and vesicles having no molecular motors on them. The proposed transport mechanism, which we call "hitchhiking", provides a consistent explanation for the broadly observed yet still mysterious phenomenon of bidirectional transport along MTs. We show that cells exploiting the hitchhiking mechanism can effectively up- and down-regulate the transport of different vesicles by tuning their binding kinetics to characteristic MT oscillation frequencies.
\end{abstract}

PACS numbers: $82.37 . R s$ 87.15.Kg 87.15.Vv 87.16.Tb

Molecular motor mediated transport along microtubules (MTs) is a well studied phenomenon in vitro. There is an increasing number of studies of classic microtubule motors like kinesin and dynein that shed light on their mechanochemistry in the idealized situation of in vitro single molecule assays [1]. Despite significant in vitro advances, understanding how intracellular transport works in vivo still remains one of the big challenges in molecular biology. Questions like how cellular cargo vesicles find their way through the cytoplasm and get targeted to their temporary or final destinations are at the heart of the problem. One of the major mysteries in this context is a phenomenon called "bidirectional transport" (BDT) [2, [3]: The majority of cargos in the cell move in a bidirectional and remarkably symmetric manner. Despite the known kinetic and dynamic asymmetry of the underlying + and - end directed motors (kinesin and dynein respectively), the vesicles seem to move with the same rates and run length distributions, and exhibit identical stalling forces, in each direction [2, 3].

Previous work on BDT has been dominated by the search for a hypothesized coupling element coordinating the actions of motors attached to a cargo vesicle to produce the observed back and forth symmetric motion. But after years of quest for the molecules that establish the predicted coupling between dynein and kinesin, the molecular mechanism of the putative coordination model [2] remains obscure. In its very basis the coordination model remains controversial, as it is hard to imagine any molecular regulator that would symmetrize the behavior of two so distinct motor species like dynein and kinesin. Nevertheless there is strong evidence from several model organisms [2, 3] that when either dynein or kinesin are impeded in their function the bidirectional transport gets affected in both + and - directions symmetrically. Here we propose a physically and biologically plausible molecular model for bidirectional transport that can account for the symmetric behavior in both directions.

Our model has two basic ingredients: 1. Motors of either or both types (not necessarily localized to the cargo) generate longitudinal MT fluctuations by already known mechanisms, e.g. the one depicted in Fig. 1a. This motor induced confined MT sliding we call "jabberwalking" of the underlying motors. While interMT shearing forces are best known for motile organelles containing the axoneme structure [4], an increasing number of studies show vigorous sliding of cytoplasmic MTs when they become detached from cellular structures [5]. Two-point microrheology studies of cytoskeletal stressstrain fluctuations that demonstrate the presence active force doublets [6] go along the same line of evidence. 2. Vesicles transiently couple to the random, stochastically oscillating MTs and attain their speed temporarily. As a consequence, the vesicles experience strongly enhanced diffusion. This process we call "hitchhiking" of the vesicles. The occurrence of such a mechanism is most clearly observed for reticulopodial cytoskeleton extensions [7] and chlamydomonas flagella [8]. In those systems, artificial and endogenous cargos (Fig. 1c) are found to move as a consequence of motor driven MT sliding in a bidirectional manner. More recently, evidence was found for hitchhiking in S2 drosophila cell lines [9] indicating a possible ubiquity of the process.

Below we first focus on the hitchhiking process and compute how a vesicle transiently coupling to a single longitudinally oscillating MT moves on long timescales (Fig. 1b). Each time the cargo binds to the MT, it assumes the latter's direction and velocity $v=v_{\mathrm{MT}}$; when it is unbound, we assume complete immobility i.e., $v=0$. We neglect here the thermal diffusion of the unbound vesicle due to its smallness compared to the active transport we consider here (see discussion below). The velocity $v_{\mathrm{MT}}(t)$ itself is a random process with the particular property that $x_{\mathrm{MT}}(t)=\int_{0}^{t} v_{\mathrm{MT}}(\tau) d \tau$ is a bounded variable, i.e., we assume the MT to move in spatially confined fashion. Consequently a cargo simply permanently sticking to the MT does not get far, and so moves in a confined manner as well. However by virtue of the switching (attachment/detachment) process, the cargo coordinate $x(t)$ can become unbounded! 


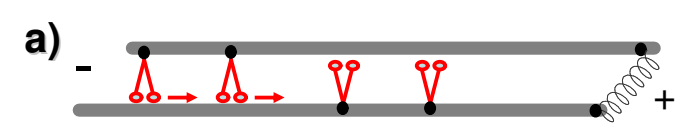

b)

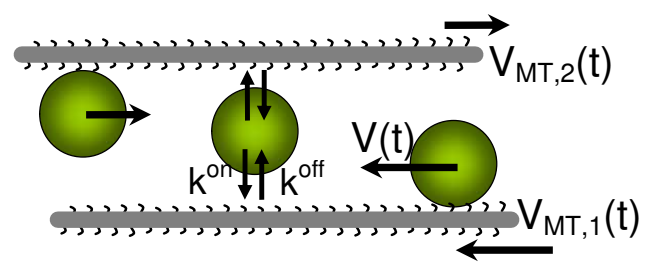

c)

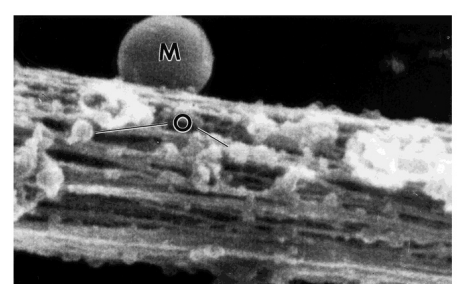

FIG. 1: a. Longitudinal MT noise caused by "jabberwalking" of double attached motors on elastically tethered MTs. $b$. The hitchhiking mechanism: Vesicle binding and unbinding kinetics, combined with local MT oscillations, induces diffusive, long range transport. MT surface polarity can also give rise to a directed drift. c. Reticulomyxa transports microspheres (M) and organelles (O) via MT sliding (adapted from []]).

The vesicle binding process is described by a 2 -state random variable $B(t) \in\{0,1\}$ with the characteristics of telegraphic noise [11]: $B(t)$ equals 1 if the cargo sticks to the MT or 0 for a detached resting cargo. We denote the stochastic switching rates between those states by $k^{\text {off }}$ and $k^{\text {on }}$. We next write the velocity of the cargo vesicle as a composite random process $v(t)=$ $B(t) v_{\mathrm{MT}}(t)$. The cargo velocity correlation function is given by $\left\langle v\left(t_{1}\right) v\left(t_{2}\right)\right\rangle=\left\langle B\left(t_{1}\right) B\left(t_{2}\right) v_{\mathrm{MT}}\left(t_{1}\right) v_{\mathrm{MT}}\left(t_{2}\right)\right\rangle$, where $\langle\cdots\rangle$ denotes the ensemble average over all realizations of $B$ and $v$. For simplicity, at first we focus on the limiting case of velocity independent rates $k^{\text {off }}$ and $k^{\text {on }}$ (we consider the general case of velocity dependence below). In this case, the vesicle binding becomes statistically independent from the $\mathrm{MT}$ motion $\left\langle B v_{\mathrm{MT}}\right\rangle=$ $\langle B\rangle\left\langle v_{\mathrm{MT}}\right\rangle, \quad B(t)$ becomes a standard (asymmetric) Markovian telegraphic noise process [11], and we easily compute $\left\langle v\left(t_{1}\right) v\left(t_{2}\right)\right\rangle=C_{\mathrm{MT}}\left(t_{1}, t_{2}\right) C_{B}\left(t_{1}-t_{2}\right)$ with $C_{\mathrm{MT}}\left(t_{1}, t_{2}\right)=\left\langle v_{\mathrm{MT}}\left(t_{1}\right) v_{\mathrm{MT}}\left(t_{2}\right)\right\rangle$ and $C_{B}\left(t_{1}-t_{2}\right)=$ $K^{2}+K(1-K) e^{-2\left|t_{1}-t_{2}\right| / t_{\mathrm{sw}}}$. The two constants $K$ and $t_{\mathrm{sw}}$ characterize the binding behavior. $K=\langle B\rangle=$ $k^{\text {on }} /\left(k^{\text {on }}+k^{\text {off }}\right)$ denotes the equilibrium binding constant and $t_{\mathrm{sw}}=2 /\left(k^{\mathrm{on}}+k^{\text {off }}\right)$ is the "mean switching time." Note that whereas the switching process $B(t)$ is assumed to be stationary in the statistical sense, the velocity $v_{\mathrm{MT}}(t)$ can in general be a non-stationary stochastic or even a purely deterministic process, and its autocorrelation function $C_{\mathrm{MT}}\left(t_{1}, t_{2}\right)$ is not necessarily time homogeneous. The mean-square-displacement (msd) of such a vesicle is obtained from

$$
\left\langle(x(t)-x(0))^{2}\right\rangle=\int_{0}^{t} \int_{0}^{t} C_{\mathrm{MT}}\left(t_{1}, t_{2}\right) C_{B}\left(t_{1}-t_{2}\right) d t_{1} d t_{2}
$$

This general expression relates the msd of the cargo to the fluctuations of a single microtubule and the binding/unbinding kinetics of the cargo to the MT. For the special case of a statistically stationary process $v_{1}(t)$, the correlator $C_{\mathrm{MT}}\left(t_{1}, t_{2}\right)=C_{\mathrm{MT}}\left(t_{1}-t_{2}\right)$ becomes homogeneous in time and we can simplify Eq.1 further

$$
\left\langle(x(t)-x(0))^{2}\right\rangle=2 \int_{0}^{t}(t-\tau) C_{B}(\tau) C_{\mathrm{MT}}(\tau) d \tau
$$

Now we illustrate our general formulas by focusing on two particular possible MT shaking processes: (1) Deterministic oscillation of the MT with $v_{M T}(t)=V \sin \omega t$, and (2) An idealization of MT motion as an overdamped harmonic oscillator driven by Markovian telegraphic noise (representing the action of motors, Fig. 1a).

Periodically oscillating $M T$. In the first case, we have $C_{\mathrm{MT}}\left(t_{1}, t_{2}\right)=V^{2} \sin \omega t_{1} \sin \omega t_{2}$, and it is easy to evaluate the rhs of Eq.11 (Eq.2 cannot be used because of the non-stationarity of $\left.v_{1}(t)\right)$. The result can be simplified in two limiting cases. On short timescales $t \ll t_{\mathrm{sw}}$, the vesicle does not have enough time to bind/unbind from the MT, and we simply have $C_{B}(t) \approx K$. From Eq.1 we obtain $\left\langle(x(t)-x(0))^{2}\right\rangle=K \omega^{-2} V^{2}(1-\cos t \omega)^{2}$, as expected for a particle strictly following the MT with probability $K$. In particular, the vesicle stays spatially confined. In the opposite limit $t \gg t_{\mathrm{sw}}$ the vesicle motion becomes diffusive: $\left\langle(x(t)-x(0))^{2}\right\rangle=$ const. $+2 D t$, with the diffusion constant given by

$$
D=K(1-K) V^{2} t_{\mathrm{sw}}\left(4+\omega^{2} t_{\mathrm{sw}}^{2}\right)^{-1}
$$

This remarkable expression says that a vesicle stochastically coupling to a periodically oscillating MT diffuses with an efficiency that depends on the fine tuning of the MT oscillation frequency $\omega$ and the stochastic vesicle switching time $t_{\mathrm{sw}}$. The long time $\left(t \gg t_{\mathrm{sw}}\right)$ transport efficiency is maximized for $K^{\mathrm{opt}}=1 / 2$ and $t_{\mathrm{sw}}^{\mathrm{opt}}=2 \omega^{-1}$, and falls off to zero away from these values. The intuitive meaning of the first result $\left(K^{\text {opt }}=1 / 2\right)$ is clear: If the vesicle sticks too strongly to the $\mathrm{MT}(K=1)$ or not at all $(K=0)$, there is no long range transport as it either moves with the MT (in a confined manner) or not at all. The optimum occurs for an intermediate value. The second finding $t_{\mathrm{sw}}^{\mathrm{opt}}=2 \omega^{-1}$, which resembles stochastic resonance phenomena [10], also has a simple interpretation: If the vesicle takes too long a ride on the $\operatorname{MT}\left(t_{\mathrm{sw}} \gg \omega^{-1}\right)$, its average displacement cancels because of the pure "back and forth" motion of the MT. If the ride is too short $\left(t_{\mathrm{sw}} \ll \omega^{-1}\right)$, a similar argument 
applies. In this rather trivial example, we already see an interesting theme: Optimal transport of vesicles requires a fine tuning of vesicle binding and MT oscillations.

MT driven by stochastic motor noise. In a second more realistic approach, we model longitudinal MT oscillations by an overdamped Langevin equation with a harmonic restoring force coming from MT attachment/confinement (Fig. 1a,b): $\xi \dot{x}_{\mathrm{MT}}=-C x_{\mathrm{MT}}+F_{\mathrm{mot}}\left(\dot{x}_{\mathrm{MT}}, t\right)$. Here $\xi$ is the MT longitudinal friction constant and $C$ the MT restoring spring constant. The actively generated motor force $F_{\mathrm{mot}}\left(\dot{x}_{\mathrm{MT}}, t\right)$ depends on the detailed motor mechanochemistry, which we effectively model by a linear force-velocity relation $v_{\text {mot }}\left(F_{\text {mot }}\right) / v_{0}=1-F_{\text {mot }} / F_{0}$ with two parameters: $v_{0}$, the maximal (zero-load) velocity, and $F_{0}$, the motor stalling force. Combining this with the Langevin equation and $\dot{x}_{\mathrm{MT}}=v_{\text {mot }}$ yields the effective equation of MT motion:

$$
\xi_{\mathrm{eff}}(t) \dot{x}_{\mathrm{MT}}=-C x_{\mathrm{MT}}+F_{0}(t)
$$

$\xi_{\text {eff }}(t)=\xi+F_{0}(t) / v_{0}(t)$ is the effective friction constant.

Eq. 4 states that the motors contribute to an effective external force $F_{0}(t)$, but also give rise to increased effective friction $\xi_{\text {eff }}>\xi$. The driving motor force $F_{0}(t)$ and velocity $v_{0}(t)$ are stochastic variables, which can switch between two values. The dynamics of this switching generally depends on the MT-motor attachment geometry. In the simplest arrangement (Fig. 1a), motors bind rigidly and run actively on both MTs in a symmetric manner. In this case $F_{0}(t)$ and $v_{0}(t)$ both switch between two values, which for simplicity we assume to be equal in magnitude but of opposite sign, i.e., $F_{0}(t)= \pm F_{0}$ and $v_{0}(t)= \pm v_{0}$ (same number of motors of same strength on both sides), which results in a time independent friction constant $\xi_{\text {eff }}=\xi+F_{0} / v_{0}$. We assume that the motors stochastically switch direction with an exponentially distributed switching time, i.e., $F_{0}(t)$ is described by symmetric Markovian telegraph noise 11] with $p\left(F_{0}(t)= \pm F_{0}\right)=1 / 2$ and $\left\langle F_{0}\left(t_{1}\right) F_{0}\left(t_{2}\right)\right\rangle=$ ${F_{0}}^{2} \exp \left(-2\left|t_{1}-t_{2}\right| / T_{p}\right)$. Here $T_{p}$ is the processivity time of the motors (average time between direction changes).

In long time limit, where $x_{\mathrm{MT}}$ in Eq.4 becomes a stationary process, we can exploit Eq.2] to evaluate the msd of the vesicle, provided that we can compute $C_{\mathrm{MT}}(\tau)=\left\langle\dot{x}_{\mathrm{MT}}(t+\tau) \dot{x}_{\mathrm{MT}}(t)\right\rangle$. To accomplish this, we use the solution of Eq. प4 in the limit $\xi_{\text {eff }}^{-1} C t \gg 1$ : $x(t)=\int_{0}^{t} \xi_{\text {eff }}^{-1} F_{0}(\tau) e^{-\xi_{\text {eff }}^{-1} C(t-\tau)} d \tau$. After some calculation, this leads to [12]:

$$
C_{\mathrm{MT}}(t)=\frac{F_{0}^{2}}{\xi_{\text {eff }}^{2}} \frac{2 T_{r} T_{p}}{4 T_{r}^{2}-T_{p}^{2}}\left(\frac{2 T_{r}}{T_{p}} e^{-\frac{2|t|}{T_{p}}}-e^{-\frac{|t|}{T_{r}}}\right)
$$

The two characteristic timescales are now the MT relaxation time $T_{r}=\xi_{\text {eff }} / C$ and the motor processivity time $T_{p}$. Inserting Eq.[5] into Eq.2] gives a lengthy expression which in the limit $t \gg \max \left(T_{p}, T_{r}, t_{\mathrm{sw}}\right)$ can be used to compute the long-time diffusion constant

$$
D=\frac{F_{0}{ }^{2}}{C^{2}} \frac{2 K(1-K) t_{\mathrm{sw}} T_{p}}{\left(2 T_{r}+t_{\mathrm{sw}}\right)\left(t_{\mathrm{sw}}+T_{p}\right)\left(T_{p}+2 T_{r}\right)}
$$

As in the previous example (periodic MT oscillation), the vesicle mobility is always maximized for $K^{\mathrm{opt}}=1 / 2$, i.e., for an intermediate binding strength. The optimal vesicle switching time $t_{\mathrm{sw}}^{\mathrm{opt}}=\sqrt{2 T_{r} T_{p}}$ is also easily obtained from Eq.6] Interestingly, for fixed $T_{r}$, Eq.6] predicts the optimal ratio $T_{p} / T_{r}=2$. For those values we obtain the maximal diffusion constant $D_{\max }=$ $F_{0}^{2}\left(\xi+F_{0} / v_{0}\right)^{-1} C^{-1} / 32$, i.e., stronger and faster motors $\left(F_{0}\right.$ and $v_{0}$ large $)$, weaker MT confinement $(C$ small $)$ and smaller MT friction give rise to more efficient transport.

Multiple MTs. For $n$ different oscillating MTs, the particle velocity is $v(t)=\sum_{k=1}^{n} \delta_{k, B(t)} v_{M T_{k}}(t)$ with $v_{M T_{k}}$ the velocity of the $k$ th MT, $\delta_{k, l}$ the Kroneker-delta and $B(t) \in\{0,1,2, . ., n\}$ the "binding variable" which indicates to which MT the vesicle is bound at time $t$ (0 represents the unbound state). For the two-MT situation in Fig. 1a, $n=2$ and $v_{M T_{1}}=-v_{M T_{2}}$ (anticorrelated MT velocities). Using Eq. [5 as before yields the resonance condition: $k^{\text {on }} \gg k^{\text {off }}, k^{\text {off }}=\sqrt{2}\left(T_{r} T_{p}\right)^{-1 / 2}$ and $T_{p}=2 T_{r}$, which differs slightly from the single MT case because it is now more favorable to jump between the two MTs than to spend time in the unbound state. The corresponding diffusion constant $D_{\max }=F_{0}^{2}\left(\xi+F_{0} / v_{0}\right)^{-1} C^{-1} / 8$ is 4 times larger than in the single MT case. This indicates that the efficiency of transport, as well as the natural strategy for optimizing it, can depend on the effective number of participating MTs.

Hitchhiking vs thermal diffusion. In the limit of motor forces larger than viscous forces $\left(F_{0} \gg \xi v_{0}\right)$, we obtain the rough estimate $D_{\max } \approx \frac{1}{8}\left(F_{0} / C\right) v_{0}$. Typical MT sliding velocities $v_{0} \approx 1-5 \mu \mathrm{m} / \mathrm{s}$ and oscillation amplitude $F_{0} / C \approx 0.1-1 \mu \mathrm{m}$ yield $D_{\max } \approx 0.01-0.5 \mu \mathrm{m}^{2} \mathrm{~s}^{-1}$. In comparison, a typical organelle with diameter $500 \mathrm{~nm}$ experiences a large cellular viscosity $\eta \approx 0.3 \mathrm{~Pa} \cdot \mathrm{s}[1]$ and has a thermal diffusion constant $D_{\text {th }} \approx 3 \cdot 10^{-3} \mu \mathrm{m}^{2} \mathrm{~s}^{-1}$. On the other hand, nanometer sized molecules experience a much smaller effective viscosity (close to that of water $\eta \approx 10^{-3} \mathrm{~Pa} \cdot \mathrm{s}$ ) and so have $D_{\text {th }} \approx 10-100 \mu \mathrm{m}^{2} \mathrm{~s}^{-1}$. Therefore large objects like vesicles and organelles can strongly benefit from hitchhiking while smaller molecules are more efficiently transported by thermal diffusion.

Hydrodynamic stress and biased hitchhiking. It is straightforward to show that the hitchhiking process as described above for large $t$ becomes an unbiased diffusive process even for an asymmetric MT shaking [9]. We consider next an interesting generalization of the hitchhiking model which gives rise to biased directed transport.

In the following, we drop the previous assumption $\left\langle B v_{\mathrm{MT}}\right\rangle=\langle B\rangle\left\langle v_{\mathrm{MT}}\right\rangle$ i.e., the statistical independence 
of the MT velocity and the vesicle-MT binding process. For example, a statistical coupling of binding and MT sliding can appear when the off rate $k^{\text {off }}\left(v_{\mathrm{MT}}\right)$ becomes velocity dependent due to a hydrodynamic drag force (with respect to the essentially stationary cytoplasm) acting on a moving vesicle. The presence of this force can break the symmetry of transport in two different ways. The general form of the off rate will be $k^{\text {off }}\left(v_{\mathrm{MT}}\right) / k^{\text {off }}(0) \approx 1+c_{2} v_{\mathrm{MT}}^{2}+c_{3} v_{\mathrm{MT}}^{3}+\cdots$. The linear term must vanish, if we make the plausible requirement that $k^{\text {off }}\left(v_{\mathrm{MT}}\right)$ is minimal for $v_{\mathrm{MT}}=0$ (no hydrodynamic stress $=$ maximal binding strength). We assume that the two remaining terms $c_{2} v_{\mathrm{MT}}^{2}$ and $c_{3} v_{\mathrm{MT}}^{3}$ are small. We call the coefficient $c_{2}$ the dynamical bias coefficient, because it gives rise to a particle drift only if the MT has different forward and backward velocity. We call $c_{3}$ the polarity bias, as it can give rise to drift even for a time reversal symmetric $v_{\mathrm{MT}}(t)$. Physically, this term stems from the polarity of MT surface and the resulting polarity of the interaction with the vesicle.

We next derive the mean drift velocity $\langle v(t)\rangle$ of the vesicle in the simplifying limiting case of rapid binding equilibration i.e., $k^{\text {on }}+k^{\text {off }} \gg \omega_{\mathrm{MT} \text {, char }}$ where $\omega_{\mathrm{MT}}$,char denotes the characteristic oscillation frequency of the MT. In this limit, the vesicle binding state equilibrates at each instant of time and the conditional binding probability becomes a function of the instantaneous MT velocity: $p\left(B=1 \mid v_{\mathrm{MT}}\right)=$ $k^{\mathrm{on}} /\left(k^{\mathrm{off}}\left(v_{\mathrm{MT}}\right)+k^{\mathrm{on}}\right) \approx K_{0}-K_{0}\left(1-K_{0}\right)\left(c_{2} v_{\mathrm{MT}}^{2}+\right.$ $\left.c_{3} v_{\mathrm{MT}}^{3}\right)$ with $K_{0}=k^{\mathrm{on}} /\left(k^{\mathrm{off}}(0)+k^{\mathrm{on}}\right)$. Consequently the vesicle's mean drift velocity simplifies to $\langle v(t)\rangle=\left\langle v_{\mathrm{MT}}(t) p\left(B=1 \mid v_{\mathrm{MT}}(t)\right)\right\rangle=\left\langle v_{\mathrm{MT}}(t)\right\rangle K_{0}-$ $K_{0}\left(1-K_{0}\right)\left(c_{2}\left\langle v_{\mathrm{MT}}^{3}(t)\right\rangle+c_{3}\left\langle v_{\mathrm{MT}}^{4}(t)\right\rangle\right.$ and its long time mean (vesicle drift) reads:

$$
\bar{v}=-K_{0}\left(1-K_{0}\right)\left(c_{2} \overline{\left\langle v_{\mathrm{MT}}^{3}\right\rangle}+c_{3} \overline{\left\langle v_{\mathrm{MT}}^{4}\right\rangle}\right)
$$

where the long time average $\bar{f} \equiv \lim _{T \rightarrow \infty} T^{-1} \int_{0}^{T} f(t) d t$. $\overline{\left\langle v_{\mathrm{MT}}\right\rangle}$ vanishes because of MT confinement.

As an example, we consider a (deterministic) asymmetric square wave MT oscillation with $v_{\mathrm{MT}}(t)$ periodically switching between $v_{\mathrm{MT}}=V_{1}$ for a time $T_{1}$ and $v_{\mathrm{MT}}=V_{2}$ for time $T_{2}$ with $V_{1} V_{2}<0$ and $T_{1} V_{1}+T_{2} V_{2}=0$ (zero mean). For such a choice from Eq. [7 we obtain $\bar{v}=-K_{0}\left(1-K_{0}\right)\left(T_{1}+T_{2}\right)^{-1}\left(c_{2}\left(T_{1} V_{1}^{3}+T_{2} V_{2}^{3}\right)+\right.$ $\left.c_{3}\left(T_{1} V_{1}^{4}+T_{2} V_{2}^{4}\right)\right)$ which in general has both the dynamical $\left(c_{2} \ldots\right)$ and the polar $\left(c_{3} \ldots\right)$ drift contributions. For a completely symmetric shaking $\left(V_{2}=-V_{1}, T_{1}=T_{2}\right)$, the dynamical part vanishes as expected, whereas the polar part always stays present with a sign opposite to $c_{3}$.

In conclusion, we have outlined a transport mechanism alternative to the standard "cargo hauled by a motor" model. Active longitudinal motions of MTs, combined with even weak non-specific cargo-MT binding, naturally lead to this transport scenario. Remarkably, even small scale (spatially confined) MT oscillations induce a long distance transport on the cellular scale. Hitchhiking on approximately symmetric MT bundle arrangements as in Fig. 1a inherently bears the feature of velocity and run-length distribution symmetry often observed in BDT 2, 3]. Very large moment velocities [3, 4, 7] (up to $>10 \mu \mathrm{m} / \mathrm{s}$ ) - another characteristic of BDT-may be attributable to the enhanced motor efficiency known for cooperatively synchronized motor batteries performing filament sliding [13] and elastic MT relaxations [9]. Other signature behaviors of our mechanism, such as visible sliding of MTs, tandem motion of vesicles, exponential velocity relaxations and strong MT bending deformations have been observed recently [7, 9]. In general, we expect a combination of hitchhiking and hauling mechanisms to be present in varying proportions for different cargos and cells. Future experiments might reveal the relative importance of their contributions and the "master plan" behind them.

We thank J. Crocker, B. Hoffman, P. Janmey, and P. Baas for discussion. We are indebted to P.R. Selvin, V. Gelfand, B. Blehm, H. Kim, and C. Kural for fruitful discussions and for sharing their movies prior to publication. This work was supported in part by NSF Grants DMR04-04674 and DMR04-25780.

* E-mail: kulic@sas.upenn.edu

[1] J. Howard, Mechanics of Motor Proteins and the Cytoskeleton. Sinauer Press (2001).

[2] S.P. Gross, M.A. Welte, S.M. Block and E.F. Wieschaus, J. Cell. Biol, 156, 715, (2002); M.A. Welte Curr. Biol. 14, R525 (2004).

[3] S.C. Ling et al. Proc. Natl. Acad. Sci. USA, 101, 17428 (2004); C. Kural et al., Science 308, 1469 (2005).

[4] L. A. Amos and W. G. Amos, Molecules of the Cytoskeletion, Guilford Press (1991).

[5] L. Wang and A. Brown, Curr. Biol 12, 1496 (2002); Y. He et al., J. Cell. Biol 168, 697 (2005); T. J. Keating et al., Proc. Natl. Acad. Sci. USA 94,5078 (1997).

[6] A.W.C. Lau et al. Phys. Rev. Lett 91, 198101 (2003).

[7] D.D. Orokos, S.S. Bowser, J.L. Travis, Cell Motil. Cytoskel. 37, 139 (1997); D.D. Orokos, R.W. Cole, J.L. Travis, Cell Motil. Cytoskel. 47, 296 (2000) and references therein.

[8] R.A. Bloodgood. J. Cell. Biol 75, 983 (1977).

[9] B. Blehm, H.Kim, C. Kural, V.I. Gelfand, P.R. Selvin, I.M. Kulić and P.C. Nelson in preparation.

[10] L. Gammaitoni, P. Hanggi, P. Jung, F. Marchesoni, Rev. Mod. Phys. 70, 223 (1998).

[11] W. Horsthemke and R. Lefever, Noise-Induced Transitions, Springer 1984.

[12] These results are valid for strongly processive motors like cytoplasmic kinesin/dynein. For a review of nonprocessive cooperative motor oscillations see F. Julicher, A. Ajdari, and J. Prost Rev. Mod. Phys. 69,1269 (1997).

[13] R. N. Seetharam and P. Satir, Cell Motil. Cytoskel. 60, 96103 (2005). 